\title{
Niños, niñas y adolescentes en situación de calle: Un estudio local Homeless Children: A local study
}

Jaime Montes

Jaime Montes Lihn es sociólogo graduado de la Universidad Católica de Chile. Actualmente es Coordinador de proyectos de la Fundación San Carlos de Maipo. Su dirección postal es Asturias 103, casa D. Las Condes y su e-mail es jmontes@focus.cl

\begin{abstract}
Resumen
Esta nota presenta las caracteristicas de los niños, niñas y adolescentes que habitan en la calle en la Región Metropolitana y discute los principales nudos críticos a resolver para la interrupción de su situación y algunas propuestas. El análisis se basa en el trabajo desarrollado por el Observatorio Metropolitano de niños, niñas y adolescentes en situación de calle, cuyo objetivo es "contribuir a dar término a la situación de vida de calle de aquellos niños/as y adolescentes que pernoctan en ella".

Palabras claves (Niños, niñas y adolescentes en situación de calle; estudio local; nudos críticos, propuestas)

Abstract

This keynote presents the characteristics of homeless children in the Metropolitan area, and discusses main critical dimensions to resolve the interruption of their situation and to develop some proposals. The analysis is based on the effort carried out by the Homeless Children's Metropolitan Observatory, which aims to "help bring an end the situation of homeless children and teenagers who have a street life".
\end{abstract}

Key words (Homeless children, local study, critical dimensions, street life interruption)

\section{Introducción}

¿Cómo lograr en el mediano plazo la reinserción social de los niños niñas y adolescentes ${ }^{1}$ en situación de calle? ¿Es posible? ¿Cuántos niños ingresan al circuito de calle cada año? ¿Cuántos niños vuelven a sus hogares? ¿Cuáles son sus características? Los niños en situación de calle, ¿son los mismos de siempre o existe un flujo de entrada y salida?

El objetivo de este artículo es dar a conocer el trabajo del Observatorio Metropolitano de niños, niñas y adolescentes en situación de calle, entregando los principales resultados arrojados y las reflexiones que han surgido en torno a esta población.

\section{Distinción entre niños "en" la calle y aquellos que "habitan en" la calle}

Es necesario distinguir entre niños en la calle y los que habitan en $^{2}$ la calle (SENAME, 2004, p.9). Los niños en la calle son aquellos que pasan una parte del día en la calle y regresan a sus casas luego de realizar algún tipo de trabajo que les reporta un ingreso para ellos y/o sus familias. No necesariamente son desertores escolares y generalmente colaboran con el ingreso familiar. Duermen en el hogar y mantienen con sus familias un vínculo directo e incluso de dependencia, pese a la autonomía que les otorga el hecho de pasar gran parte del tiempo en la calle.

Los niños que habitan en la calle son aquellos cuyos vínculos familiares están debilitados, al punto que viven esencialmente en la calle, dependiendo de sus propios esfuerzos para cubrir todas sus necesidades básicas. Su situación de marginación los coloca en un riesgo más severo que la mayoría de los otros niños y niñas. La ruptura del vínculo con su familia, la cual vive por lo general en la pobreza, constituye el punto de partida del proceso que lleva al niño a transformarse en niño que habite en la

1 Niños, niñas y adolescentes: en adelante, niños.

2 En el estudio de SENAME (2004), se hace la distinción de niño "en" la calle y niño "de" la calle. Sin embargo, considero más pertinente cambiar la palabra "de" por "habita en", para realzar que es una situación y no una condición. 
calle. Teniendo y reconociendo tener familia, se han separado de la misma, organizando su vida y hogar en la calle. El niño que habita en la calle usa este espacio de diversas formas y permanece en ella en forma esporádica o permanente. Sus condiciones de vida están caracterizadas por el trabajo prematuro, la baja escolaridad o analfabetismo, la desvinculación o distanciamiento de la familia, experiencias de explotación sexual, conductas infractoras de la ley y pobreza. Su perfil está asociado a su condición de desertores escolares, provienen de familias en extrema pobreza, cuyos padres son por lo general cesantes o desarrollan trabajos precarios, tienen escaso contacto con redes de apoyo social y una importante mayoría ha recibido atención en los centros del Servicio Nacional de Menores (SENAME).

Si bien la situación de calle es un continuo entre estas dos distinciones, esta separación es útil para enfocarse en aquellos niños que reúnen las condiciones de mayor vulnerabilidad en sus derechos.

En adelante, la población a las que nos referiremos es la de aquellos niños que habitan en la calle, específicamente en la Región Metropolitana.

\section{¿Cuántos son? ¿Son los mismos de siempre o existe un gran flujo de entrada y salida de la situación de calle?}

Hasta ahora han existido estudios transversales que han sido de gran aporte para dimensionar el fenómeno en Chile. Según el Catastro Nacional de personas en situación de calle realizado el año 2005 por MIDEPLAN, existen en Chile 674 niños, niñas y adolescentes que habitan en la calle, lo que corresponde a un 9,3\% del total de 7.254 personas que se encuentran en esta situación. De estos niños, 322 viven en la Región Metropolitana, lo que significa que esta zona concentra cerca de la mitad de esta población. Entre ellos, 118 tendrían entre 14 y 17 años y 204 serían menores de 14 años. Estos datos entregan un primer nivel de información, pero dejan muchas interrogantes en cuanto a la dinámica de esta población.

Los niños, niñas y adolescentes que habitan la calle conforman una población flotante. Un niño, niña o adolescente no pasa a vivir en la calle de un día para otro, el fenómeno involucra un proceso que puede durar hasta un año. En este sentido resulta difícil obtener una cuantificación absoluta y certera de este grupo dado que la calle es un espacio por el cual ellos transitan de manera itinerante (con entradas y salidas). Además, para pernoctar, algunos varían entre el uso de hospederías y la vía pública, y varios de ellos van de vez en cuando a pasar unos días en sus hogares. Por su parte, las mismas caletas en las que viven son espacios que no tienen mayor relación con un lugar fijo de permanencia, más bien se trata de grupos rotativos de personas. Asimismo existirían niños que deambulan generalmente solos, sin conformar caletas, lo que aumenta la dificultad para detectarlos.

Por iniciativa de la Fundación San Carlos de Maipo, se ha convocado el Observatorio Metropolitano de niños, niñas y adolescentes en situación de calle, para avanzar en su objetivo de contribuir a dar término a la situación de vida de calle de aquellos niños/as y adolescentes que pernoctan en ella en la Región Metropolitana. El Observatorio cuenta con un Consejo formado por representantes del estado, la sociedad civil y el mundo académico: Hogar de Cristo, Fundación Don Bosco - Vida Compartida, SENAME, CONACE, el Programa de Políticas Públicas de la Pontificia Universidad Católica de Chile y la Fundación San Carlos de Maipo.

Para dar cumplimiento al objetivo, el primer paso es conocer la situación de cada uno de los niños. Por medio del esfuerzo de las instituciones que trabajan directamente con ellos, se identificó a cada uno de los niños en un sistema de registro y detección temprana. Se incluyeron no solamente los niños atendidos bajo financiamiento del SENAME, sino además otros que no están siendo atendidos por las instituciones especializadas, pero sí han sido contactados por ellas. El sistema de registro permite no solamente saber cuántos son, sino cuál es el avance en el proceso de interrupción de la situación de calle de cada uno de ellos. No fue una tarea fácil pues hay niños que solo son conocidos por el apodo, $u$ otros de los que no se conoce su RUT. Por ello, se establecieron requisitos mínimos que permitieran tener información confiable, evitando la duplicación de los datos. Este sistema permite hacer estudios longitudinales para determinar los flujos de entrada y salida a la situación de calle, un punto crucial no solamente para una mejor comprensión del fenómeno sino también para tomar medidas tendientes a frenar tempranamente el ingreso al circuito de calle.

El sistema de información permite, primero, detectar tempranamente el ingreso de un niño, niña o adolescente a la situación de calle y de esta forma promover su atención a la brevedad. Segundo, al ser un registro al cual podrán acceder las instituciones que trabajan con esta población se fomentará una intervención coordinada en donde el niño, niña o adolescente tendrá la posibilidad de seguir un pro- 
ceso coherente que le permita salir definitivamente de su situación de calle. Tercero, permitirá conocer con exactitud la población objetivo total, ya que una dificultad importante en la implementación de los programas es que es una población flotante y que no ha sido identificada del todo.

Aún no es posible contar con estudios longitudinales, ya que el informe que analizaremos a continuación es el primero, y data de diciembre de 2007. Según estos datos, en la Región Metropolitana existen 308 niños, niñas y adolescentes que habitan en la calle; diecisiete de ellos cumplieron 18 años durante el 2007, por lo tanto la población menor de 18 años es de 291 niños en situación de calle en la Región Metropolitana.

Contribuir a la interrupción de la situación de calle es una tarea sin duda desafiante, y que requiere del esfuerzo mancomunado de todos los sectores de la sociedad. Algunos niños que habitan en la calle presentan de manera conjunta problemas sociales como el consumo abusivo de drogas, enfermedades mentales, interrupción prolongada de la escolarización o conductas de infracción a la ley. Un porcentaje importante de ellos cuenta con una relación precaria con la familia y la escuela, siendo éstos los principales agentes en su desarrollo. Sin embargo, esta población también cuenta con enormes potencialidades y habilidades individuales que no siempre son visualizadas producto de la grave estigmatización que sufren.

Por otro lado, representan un problema menor en términos numéricos para las políticas públicas ya que no son más de mil en Chile. No obstante aque- llo, configuran una combinación de factores de riesgo que los posiciona entre los más vulnerados; por lo tanto, actuar decididamente para contribuir al término de su situación de calle se convierte en un imperativo ético, sobre todo si ya están individualizados y el tiempo en calle va deteriorando día a día su situación y vulnerando gravemente sus derechos.

La propuesta del Observatorio en este sentido es crear un mecanismo que minimice la entrada a los niños al circuito de calle, mediante un eficaz sistema de detección temprana que corte el flujo de ingreso de manera efectiva. Se sabe que a mayor tiempo en calle, es más improbable que el niño regrese a su familia o viva de manera autónoma. En otras palabras, a mayor tiempo en calle, el niño se apropia de la cultura callejera y es probable que siga habitando en la calle hasta la edad adulta. Por ello, es fundamental que la detección temprana sea efectiva para realizar un trabajo psicosocial cuando los niños llevan el mínimo de tiempo en calle combinado con una atención especializada a la familia de origen, la familia extendida o un adulto significativo en caso que la primera no lo pueda acoger.

\section{¿Quiénes son? Descripción por sexo, edad y distribución territorial}

Los datos del Observatorio han arrojado importantes novedades respecto a la composición de la población de niños en situación de calle en la Región Metropolitana.

En primer lugar, llama la atención un importante grupo menor de 10 años y el número de niñas, como lo muestra el siguiente cuadro:

\section{RANGO DE EDAD DE LOS NIÑOS, NIÑAS Y ADOLESCENTES QUE HABITAN EN LA CALLE EN LA REGIÓN METROPOLITANA}

\begin{tabular}{|c|c|c|c|c|c|}
\hline \multirow{2}{*}{ Rango de edad } & \multicolumn{2}{|c|}{ Sexo } & Total general & Porcentaje & $\begin{array}{c}\text { Porcentaje } \\
\text { acumulado }\end{array}$ \\
\cline { 2 - 3 } & Femenino & 16 & 28 & $9,6 \%$ & $14,6 \%$ \\
\hline $\begin{array}{c}\text { Rango A: } \\
\text { 0 a 5 años }\end{array}$ & 12 & 42 & 51 & $17,5 \%$ & $27,1 \%$ \\
\hline $\begin{array}{c}\text { Rango B: } \\
6 \text { a } 10 \text { años }\end{array}$ & 9 & 101 & 126 & $43,3 \%$ & $70,3 \%$ \\
\hline $\begin{array}{c}\text { Rango C: } \\
11 \text { a } 15 \text { años }\end{array}$ & 25 & 61 & 86 & $29,6 \%$ & $100,0 \%$ \\
\hline $\begin{array}{c}\text { Rango D: } \\
16 \text { a } 18 \text { años }\end{array}$ & 25 & 220 & 291 & & \\
\hline Total general & 71 & & & \\
\hline
\end{tabular}

Fuente: Programa de Políticas Públicas UC. (Diciembre 2007). Informe mensual Observatorio Metropolitano de niños, niñas y adolescentes que habitan en la calle en la Región Metropolitana. 
El perfil de los niños en situación de calle se asocia generalmente a un adolescente de alrededor de 14 años de sexo masculino, lo cual corresponde con las cifras. Sin embargo existe una importante proporción de la población que rompe con este estándar: el $27 \%$ presenta una edad menor o igual a 10 años, lo cual en opinión de los miembros del Observatorio sería un fenómeno nuevo.

En este punto se puede hacer un paralelo con los niños que sufren explotación sexual comercial en la Región Metropolitana (Camus, 2007, p14). Se ha identificado que un $22 \%$ de la población de niños atendidos por las instituciones especializadas en explotación sexual comercial en la Región Metropolitana, es menor de 13 años. Las ONG participantes en el estudio han manifestado también el aumento en los últimos años de niños de menor edad en esta situación.

Esta situación debe llamar a la reflexión de los actores públicos y de la sociedad civil. ¿Cuál es la causa de este aumento de niños de corta edad en situación de calle? ¿Existe una atención especializada para este perfil de niños con una edad promedio menor y sus familias?

En cuanto al sexo, el 24\% de la población son niñas, las cuales presentan características e intereses distintos a los niños y jóvenes de sexo masculino. Esta situación también presenta un gran desafío a la intervención psicosocial y a las políticas públicas, pues si bien existe atención a las niñas, históricamente los programas especializados en calle han sido de un carácter masculino. Al igual que en el caso de los niños y niñas menores de 10 años, existe la necesidad de una mayor especialización en el caso de las mujeres. Según la opinión de los programas especializados, ellas serían más propensas que los hombres a un lugar protegido como un albergue. Actualmente en la Región Metropolitana, existen 10 cupos para albergue infanto juvenil para niños en situación de calle.

En cuanto a la distribución territorial de los niños en situación de calle en la Región Metropolitana, el Cuadro $\mathrm{N}^{\circ} 2$ muestra en detalle las características de cada comuna:

CUADRO No 2

\section{COMUNA DE LOCALIZACIÓN DE LOS NIÑOS, NIÑAS Y ADOLESCENTES QUE HABITAN EN LA CALLE EN LA REGIÓN METROPOLITANA}

\begin{tabular}{|l|c|c|}
\hline \multicolumn{2}{|c|}{ Niños y niñas por comuna } \\
\hline Comuna & Cantidad & Porcentaje \\
\hline Santiago & 97 & $33,3 \%$ \\
\hline Puente Alto & 46 & $15,8 \%$ \\
\hline Renca & 32 & $11,0 \%$ \\
\hline La Florida & 31 & $10,7 \%$ \\
\hline Estación Central & 17 & $5,8 \%$ \\
\hline Recoleta & 14 & $4,8 \%$ \\
\hline El Bosque & 12 & $4,1 \%$ \\
\hline Providencia & 8 & $2,7 \%$ \\
\hline La Pintana & 7 & $2,4 \%$ \\
\hline San Ramón & 7 & $2,4 \%$ \\
\hline Maipú & 5 & $1,7 \%$ \\
\hline La Cisterna & 3 & $1,0 \%$ \\
\hline Lo Espejo & 3 & $1,0 \%$ \\
\hline Peñalolén & 2 & $0,7 \%$ \\
\hline Colina & 1 & $0,3 \%$ \\
\hline Pedro Aguirre Cerda & 1 & $0,3 \%$ \\
\hline Talagante & $\mathbf{2 9 1}$ & $0,3 \%$ \\
\hline (Desconocida) & & $1,4 \%$ \\
\hline Total general & $100 \%$ \\
\hline
\end{tabular}

Fuente: Programa de Políticas Públicas UC. (Diciembre 2007). Informe mensual Observatorio Metropolitano de niños, niñas y adolescentes que habitan en la calle en la Región Metropolitana. 
Los datos presentes en la tabla refieren a las comunas en que el niño fue contactado. Sin embargo este dato no coincide necesariamente con la comuna de origen del niño, es decir la comuna en la que se encuentra su familia.

Las tres comunas que presentan más niños que habitan en la calle son Santiago (97 casos), Puente Alto (46 casos) y Renca (32 casos), con el 33\%, $15,8 \%$ y $11 \%$ respectivamente del total de la Región Metropolitana.

Si se agrupan por sectores, se pueden identificar tres focos principales, por una parte un centro que abarcaría las comunas de Santiago y Estación Central, las cuales concentran al 39,1\% de los niños y niñas. En segundo lugar un foco sur que abarca Puente Alto y la Florida, con el 26,5\% del total; y finalmente la zona norte Renca y Recoleta, con el $15,8 \%$. Esto se puede visualizar con mayor claridad en la siguiente figura $\mathrm{N}^{\circ} 1$.

Es interesante destacar que es la primera vez que un estudio señala la localización por comuna de los niños en situación de calle, lo cual puede dar señales para focalizar los esfuerzos de las instituciones y las políticas públicas que se tomen al respecto.

En cuanto a la cobertura de atención, actualmente, existen tres proyectos especializados de calle (PEC) financiados por el SENAME en la Región Metropolitana, los cuales se distribuyen de la siguiente forma:

\section{COMUNA DE LOCALIZACIÓN DE LOS NIÑOS, NIÑAS Y ADOLESCENTES QUE HABITAN EN LA CALLE EN LA REGIÓN METROPOLITANA}

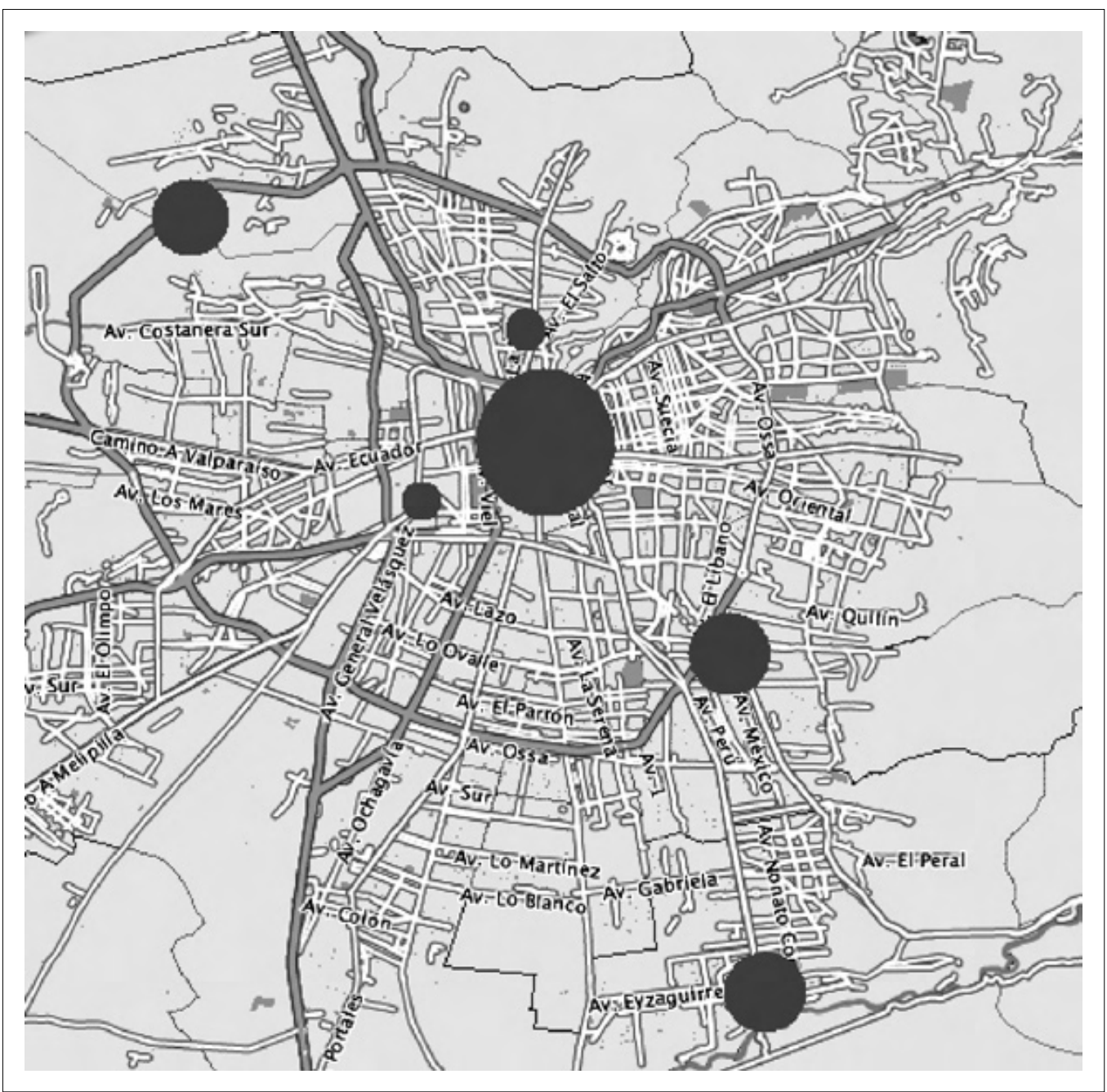

Fuente: Programa de Políticas Públicas UC. (Diciembre 2007). Informe mensual Observatorio Metropolitano de niños, niñas y adolescentes que habitan en la calle en la Región Metropolitana. 
- Existen 70 cupos para la zona Santiago, que abarca las comunas de Santiago, San Miguel, Pedro Aguirre Cerda, Maipú y Cerrillos, atendidos por la Fundación Don Bosco - Vida Compartida.

- 50 cupos para la zona de Puente Alto que comprende las comunas de Puente Alto, San Bernardo, La Pintana y Peñalolén, atendidos por la Fundación Don Bosco - Vida Compartida y

- 50 cupos para la zona de La Florida, que abarca las comunas de La Florida, La Granja y La Pintana, atendidos por Servicio Paz y Justicia (SERPAJ).

El total de niños que habitan en la calle atendidos bajo esta modalidad de atención diurna suman $120^{3}$. A esto se suman los 10 cupos para albergue, más 26 cupos que cuentan con atención diurna y nocturna atendidos por la Fundación Don Bosco Vida Compartida.

\section{Principales nudos críticos y propuesta}

Cobertura en zona norte. A pesar del esfuerzo por aumentar el número de cupos, no se abarca aún a la población total, especialmente en la zona norte de la Región Metropolitana, que no contempla proyectos especializados. Renca es la tercera comuna que presenta más niños que habitan en la calle (32 casos), y si se suma otra comuna de la zona norte, como Recoleta, la suma de ambas asiente a 46 casos.

Menores de 10 años. Los datos del Observatorio indican que el $27 \%$ de los niños y niñas son menores de 10 años (79). Este grupo se constituye como una oportunidad de intervención ya que llevarían relativamente poco tiempo en la calle, lo que hace prioritario una atención temprana que tenga mayores posibilidades de reinserción de los niños y niñas.

Niñas. Un foco en el cual es necesario poner especial atención, y en el cual aún no existe una especialización suficiente en la oferta son las niñas, quienes representan al 24\% de la población identificada. Es necesario fomentar la existencia de instancias y acciones que den respuesta a las particularidades de este grupo. Esta población está presente de manera especial en la comuna de Santiago, donde se ubican 28 de 50 niñas mayores de 11 años.

Desintoxicación de drogas. Actualmente no existe oferta pública para niños ni niñas menores de 14 años que requieran internación para desintoxica- ción de drogas, paso que es fundamental para su proceso de reinserción social. El consumo problemático de drogas es habitual en esta población.

Salud Mental. En la Región Metropolitana existen alrededor de 12 cupos para internación para patologías psiquiátricas o trastornos de personalidad grave en el Hospital Roberto del Río. Estos 12 cupos están disponibles para toda la población infantil de la Región Metropolitana (no solamente los niños que habitan en la calle) por lo cual existe también un déficit considerable.

Patología Dual. Para aquellos niños y niñas que presentan consumo problemático de drogas sumado a trastorno de personalidad grave o patología psiquiátrica no existe oferta pública para su tratamiento, debido a que no son recibidos en los cupos de salud mental. Gran parte de los casos de los niños que habitan en la calle, los temas de droga y salud mental están íntimamente relacionados, por lo cual existe un vacío de atención hacia esta población.

\section{Propuestas}

A partir de los datos arrojados por el Observatorio se puede concluir que para interrumpir la situación de calle de esta población es prioritario abrir cupos de atención en la zona norte para al menos 46 niños y niñas, darle una importancia y urgencia a la atención de niños menores de 10 años y a las niñas.

Por otro lado, a través de las reflexiones de los miembros del consejo del Observatorio es posible concluir que deberían abrirse centros de internación para desintoxicación de drogas, el aumento de los cupos para la población infantil ante problemas graves de salud mental, y la apertura de un centro de atención para aquellos niños que presentan patologías duales, es decir, consumo problemático de drogas sumado a trastorno de personalidad grave o patología psiquiátrica.

\section{Bibliografía}

CAMUS, M. J. (2007). Estudio Linea de Base de Programas contra la explotación sexual comercial infantil en la RM. Fundación San Carlos de Maipo. 2007. (Sin publicar).

PROGRAMA DE POLÍTICAS PÚBLICAS UC (2007). Informe diciembre 2007 Observatorio Metropolitano de niños, niñas y adolescentes que habitan en la calle en la Región Metropolitana. (Sin publicar).

SENAME (2004). Niños y Niñas de la Calle. Serie Estudios y Seminarios.

3 Esta cifra es el resultado de la suma de los cupos de la zona de Santiago y Puente Alto. No se contabilizan los cupos atendidos por SERPAJ, debido a que dirigen su atención al perfil de niños "en" calle. Por lo tanto, no son parte del universo de este estudio. 